\title{
Erratum to: Proximity Effect on the Density of States and Majorana Bound States in a Thin Superconducting Ferromagnetic Nanowire
}

\author{
E. R. De Lascio ${ }^{1}$ D. R. Cornejo ${ }^{1}$
}

Published online: 26 November 2016

(c) Springer Science+Business Media New York 2016

\section{Erratum to: J Supercond Nov Magn (2016) 29:2777-2782}

DOI 10.1007/s10948-016-3653-5

The original version of this article unfortunately contained three minor text errors. The sets of points satisfying the conditions $\mathrm{r} 0=0$ and $\mathrm{r} 3=\mathrm{i} 3=0$ are branch points of the Green function, not poles as is written in the text. Therefore these points do not represent resonances or bound states. They were not effectively considered in the analysis and do not have any impact on the conclusions.

The sentences:

"If $\mathrm{r} 0=0$, there is a pole, representing a resonance in $\mathrm{N}\left(\mathrm{e}^{\prime}\right)$ ". (page 3);

“. $\ldots$ or $\mathrm{r} 0=0$. For e' $>1$ it must hold $\mathrm{r} 3=\mathrm{i} 3=0$ in order to be a pole." (page 4);

“... (including that for $\mathrm{r} 0$ )" (page 5).

should be removed to make the text correct.

The online version of the original article can be found at http://dx.doi.org/10.1007/s10948-016-3653-5

E. R. De Lascio

lascio@if.usp.br

D. R. Cornejo

cornejo@if.usp.br

1 Instituto de Física, Universidade de São Paulo, São Paulo, SP, 05508-900, Brazil

These should be considered mere text errors (due to a failure in the revision of a preliminary version of the text). Therefore, the simple omission of the sentences mentioned above is enough to correct the article. 